\title{
Acortamiento de la longitud telomérica en adultos jóvenes coin
} catarata

\section{Telomere length shortening associated with cataract in
adults Fernando Cruz-González ${ }^{1,2}$, Emiliano Hernández-Galilea ${ }^{1,2}$ y Rogelio González-Sarmiento ${ }^{2,3,4}$}

${ }^{1}$ Departamento de Oftalmología, Hospital Universitario de Salamanca; ${ }^{2}$ Instituto de Investigaciones Biomédicas de Salamanca (IBSAL), Ho\$Spital Universitario de Salamanca, Universidad de Salamanca, Consejo Superior de Investigaciones Científicas (CSIC); ${ }^{3}$ Unidad de Medicina Molecular, Departamento de Medicina, Universidad de Salamanca; ${ }^{4}$ nstituto de Biología Molecular y Celular del Cáncer (IBMCC), Universidad de Salamannca, CSIC. Salamanca, España

\section{Resumen}

Objetivo: Se realizó un estudio prospectivo observacional con 205 adultos (102 casos y 103 controles) clasificados en grupos de $\leq 55$ años $y \geq 60$ años, con el fin de evaluar la longitud telomérica en pacientes con y sin catarata, y los eféctos de los polimorfismos (TERC y TERT) de la telomerasa sobre dicha longitud. Métodos: La longitud telomérica y los polimorfismos de la telomerasa se cuantificaron en leucocitos de sangre periférica por reacción en cadena de la polimerasa. Se utilizaron sondas TaqMan ${ }^{\oplus}$ para genotipificar polimorfismos de telomerasa (TERT 1327C>T yTERC 63G>A). Resultados: Independientemente de la edad, los pacientes con catarata tenían longitudes teloméricas más cortas que los controles ( $p<0.01)$. No se detectó asociación entre el acortamiento de los telómeros y TERC 63G>A en casos ni en controles, y no hubo cōrrelación entre la longitud telomérica en los leucocitos y los factores de riesgo cardiovascular en los pacientes con catarata. Conclusiones: Sin que influyera la edad, los telómeros en leucocitos de sangre periférica de pacientes con catarata fueron más cortos que en aquellos sin catarata. Nuestros hallazgos sugieren que el desarrollo de cataratas está asociado con el envejecimiento de las células de la sangre periférica.

Palabras clave: Cataratas. Diabetes. Hipertensión. Longitud telomérica. Polimorfismo. Tabaquismo.

\section{Abstract}

Purpose: A prospective observational study was performed that included 205 adults, 102 cases and 103 controls, classified into the age groups of $\leq 55$ and $\geq 60$ years old, to examine the prognostic value of telomere length in patients with or without cataract, and to assess the effects of telomerase ribonucleic acid (RNA) gene variants (TERC and TERT) on telomere length. Methods: Telomere length and telomerase polymorphisms were quantified in peripheral blood leukocytes by quantitative polymerase chain reaction. TaqMan ${ }^{\circledR}$ probes were used for telomerase polymorphisms (TERT 1327C>T and TERC 63G>A) genotyping. Results: Regardless of age, patients with cataract had shorter telomere lengths compared to healthy controls $(p<0.01)$. No association was detected between telomere shortening and TERC 63G>A in both cases and controls, and ho correlation was observed between leukocyte telomere length and cardiovascular risk factors in patients with cataracts.

Fecha de recepción: 05-03-2020 28031 Madrid, España E-mail: v.rivero.g@ hotmail.com (http://creativecommons.org/licenses/by-nc-nd/4.0/). 
Conclusions: Irrespective of age, telomeres in peripheral blood leukocytes of patients with cataract were shorter compared to subjects without cataract. Our findings suggest that the development of cataract is associated with peripheral blood $c$ ell aging.

Key words: Cataract. Diabetes. Hypertension. Telomere length. Polymorphism. Smoking.

\section{Introducción}

Los telómeros son secuencias repetidas de nucleótidos que cubren los extremos de los cromosomas. Con cada división celular, los telómeros se acortan hasta una longitud crítica cuando la célula ya no puede replicarse, provocando senescencia o muerte. Este mecanismo conduce a una disminución de la función celular. Se ha sugerido que los telómeros cortos podrían ser marcadores de baja resistencia al estrés ${ }^{1}$. En consecuencia, la longitud de los telómeros se ha propuesto como un indicador del envejecimiento celular y de la longevidad, y se ha asociado con muchas enfermedades relacionadas con la edad avanzada y la esperanza de vida ${ }^{2,3}$.

Para mantener la longitud de los telómeros, la enzima telomerasa se encarga de sintetizar las repeticiones de GGTTAG en los extremos de los cromosomas. La telomerasa humana se compone principalmente de transcriptasa inversa de telomerasa (TERT), ácido ribonucleico de telomerasa (TERC) y disquerina (DKC1). Las variantes genéticas comunes, TERT 1327C>T rs27350940 y TERC 63G>A rs2293607, se han relacionado con una actividad reducida de la telomerasa que causa un acortamiento prematuro de los telómeros ${ }^{4,5}$. Estos genes se expresan en tejidos embrionarios o células mononucleares periféricas, pero no se han identificado en el cristalino humano.

Además, la longitud de los telómeros se ha asociado con factores de riesgo cardiovascular, como diabetes, hipertensión arterial y tabaquismo ${ }^{6}$. De hecho, los pacientes con diabetes tienen telómeros más cortos que los individuos sanos ${ }^{7}$, y los individuos con longitudes teloméricas cortas muestran más complicaciones por diabetes ${ }^{8}$. Además, varios estudios han detectado un acortamiento más rápido de los telómeros en fumadores, probablemente debido al daño oxidativo causado por el tabaco ${ }^{9}$. En el Framingham Heart Study se encontró que los hombres hipertensos tenían longitudes de telómeros de leucocitos ajustadas por la edad significativamente más cortas en comparación con sus pares normotensos ${ }^{10}$. Sin embargo, esta asociación no pudo confirmarse en una cohorte mixta de hombres y mujeres examinada en el estudio de salud cardiovascular $^{11}$. Por lo tanto, podría haber un efecto relacionado con el sexo sobre la longitud de los telómeros de los leucocitos en humanos por el que la longitud delos telómeros sea más corta en los hombres adultos que en las mujeres ${ }^{12}$.

La hipótesis de que la transparencia del cristalino podría estar relacionada con la longitud de los telợneros de los leucocitos como marcador del envejecimiento está respaldada por observaciones en trastornos del envejecimiento prematuro, como el síndrome de Werner. En los pacientes con este síndrome, las características relacionadas con el envejecimiento normal, como el cáncer y las cataratas bilaterales, aparécen tempranamente, y los pacientes muestran un acortamiento acelerado de los telómeros ${ }^{13-15}$.

El presente estudio fue diseñado para examinar la Tongitud de los telómeros leucocitarios en adultos jóvenes con cataratas y evaluar la supuesta asociación entrélos polimorfismos de los genes TERT y TERC y las cataratas prematuras. También se determinó si las longitudes de los telómeros diferían entre sujetos con y sin factores de riesgo cardiovascular para eliminar los factores de confusión (tabaquismo, diabetes e hipertensión) que posibiblemente causan acortamiento de los telómeros.

\section{Método}

\section{Sujetos}

Se recolectaron muestras de sangre de 205 sujêtos después de obtener su consentimiento informado. EI protocolo del estudio recibió la aprobación del Comité de Revisión Institucional y se adhirió a los principios de la Declaración de Helsinki. Los sujetos se dividieron en cuatro grupos: 46 con catarata $\leq 55$ años emparejados con 47 sin catarata de edad similar, y 56 con catarata $\geq 60$ años emparejados con 56 sin catarata de edad similar. Las características clínicas y dempgráficas se resumen en la tabla 1. Las edades megias de los sujetos en los cuatro grupos fueron: $48.5 \pm \frac{7}{4} 47$ años (catarata $\leq 55), 43.8 \pm 7.63$ (sin catarata $\lesseqgtr 55$ años), $72.5 \pm 7.6$ años (catarata $\geq 60$ años) y $66.5 \pm$ 4.3 años (sin catarata $\geq 60$ años).

Todos los participantes se sometieron a un examen oftalmológico estándar, que incluyó medición de la agudeza visual mejor corregida (AVMC), refraceción, medición de la presión intraocular y biometría por 
Tabla 1. Características clínicas y demográficas de los sujetos incluidos en el studio

\begin{tabular}{|l|c|c|c|c|}
\hline & Casos $\leq 55$ años, $n(\%)$ & Controles $\leq 55$ años, n (\%) & Casos $\geq 60$ años, n (\%) & Controles $\geq 60$ años \\
\hline $\begin{array}{l}\text { Tamaño de la muestra } \\
\text { Sexo }\end{array}$ & 46 & 47 & 56 & 56 \\
$\begin{array}{l}\text { Hombres } \\
\text { Mujeres }\end{array}$ & $28(60.9 \%)$ & $21(44.7 \%)$ & $25(44.6 \%)$ & $18(32.1 \%)$ \\
\hline Hipertensión & $18(39.1 \%)$ & $26(55.3 \%)$ & $31(55.4 \%)$ & $38(67.9 \%)$ \\
\hline Diabetes & $9(19.6 \%)$ & $4(8.5 \%)$ & $32(57.1 \%)$ & $30(53.6 \%)$ \\
\hline Tabaquismo & $10(21.7 \%)$ & $7(14.9 \%)$ & $10(17.9 \%)$ & $13(23.2 \%)$ \\
\hline
\end{tabular}

ultrasonido. El examen también incluyó la observación del segmento anterior dilatado utilizando una lámpara de hendidura para verificar la presencia o ausencia de catarata (nuclear, cortical, subcapsular posterior) y revisar el fondo de ojo. La transparencia del cristalino se clasificó de acuerdo con el Sistema de Clasificación de Opacidades del Cristalino (LOCS) III validado.

Los criterios de inclusión de los pacientes fueron: AVMC $<0.5$ Snellen de escala decimal, una vez descartada cualquier otra patología oftalmológica que pudiera justificar la pérdida de agudeza visual, y la presencia de catarata en el examen oftalmológico. $Y$ para los controles fueron: AVMC de 1 en ausencia de catarata según el LOCS III. Todos los sujetos, casos y controles, cumplieron los mismos criterios en términos de lugar de residencia o trabajo al aire libre o en el interior (para controlar los riesgos ambientales, como la exposición al sol).

Los criterios de exclusión fueron tener tratamiento presente o pasado relacionado con el desarrollo de cataratas (corticosteroides, cirugía vitreorretiniana, quimioterapia, radiación), abuso de alcohol o cualquier enfermedad ocular (trauma, uveítis, glaucoma) o sistémica asociada con cataratas (enfermedad de Steinert, trastornos metabólicos, síndrome de Marfan y otras enfermedades del músculo esquelético).

\section{Extracción de ADN}

Se extrajo ADN genómico de células sanguíneas periféricas nucleadas siguiendo el método de fenol-cloroformo, como se informó anteriormente ${ }^{16}$.

\section{Análisis de la longitud telomérica}

La longitud de los telómeros se midió mediante reacción en cadena de la polimerasa (PCR) cuantitativa relativa y comparativa. Se comparó el número medio de copias de la secuencia de repetición de los telónneros con el gen de copia única de referencia 36B4. Para cada muestra de ADN se realizaron seis experimeñtos diferentes: tres para secuencia de repetición de telómeros y tres para gen de copia única. El método fue descrito por Gil y Coetzer en $2004^{17}$ utilizando los mismos cebadores desarrollados por Cawthon en 2002 para la longitud de los telómeros ${ }^{18}$ y el gen 36B4 cömo control endógeno ${ }^{19}$. Los cebadores específicos de tẹlómeros fueron directo (forward, hacia adelanute), 5'-GGTTTTTGAGGGTGAGGGTGAGGGTGA GGG TGAGGGT-3', e inverso (reverse, hacia atrás), 5'-TCCCGACTATCCCTATCCCTATCCCTATCCC TATCCCTA-3'. Los cebadores específicos de 36b4 fueron directo, 5'-CAGCAAGTGGGAAGG TGTAATCE-3', e inverso, 5'-CCC ATTCTATCATCAACGGGTACAA-3'20. El gen de control 36B4 se amplificó en las mișmas condiciones de PCR que el gen telomérico, reduciendo así el riesgo de variación entre placas.

Se agregó lo siguiente a cada pocillo: $5 \mathrm{ng} / \mu \mathbb{P}$ de ADN, $5 \mathrm{ml}$ de SYBR Green PCR Master Mix (Kapa Biosystems) y $4 \mathrm{ml}$ de agua. También se añadieron'1 $\mu \mathrm{l}$ de cebador directo $(5 \mu \mathrm{M})$ y $2.33 \mu$ l de cebador inverso $(5 \mu \mathrm{M})$ para la reacción de amplificación de telómeros, y $0.4 \mu \mathrm{l}(5 \mu \mathrm{M})$ de cada cebador para la amplificación del gen 36B4. El perfil de ciclos térmicos fue de la siguiente manera: 10 minutos a $95{ }^{\circ} \mathrm{C}$ seguido $\mathrm{e}$ de 35 ciclos de $95{ }^{\circ} \mathrm{C}$ durante 15 segundos, $54{ }^{\circ} \mathrm{C}$ durante 2 minutos y $72{ }^{\circ} \mathrm{C}$ durante 15 segundos $^{21}$. La ampulificación de qPCR se realizó utilizando el sistema de $\mathrm{PCR}$ en tiempo real Step-One Plus (Applied Biosystems). Para determinar la longitud relativa délos telómeros se utilizó la versión 1.2 del software $R Q$ Manager.

El valor de $\mathrm{Ct}$, definido como el número de ciclos de PCR necesarios para que el ADN amplificado cruce un 
Tabla 2. Secuencias de las sondas VIC y FAM $\left(5^{\prime}-3^{\prime}\right)$ utilizadas para la detección de los polimorfismos TERT-1327C>y TERC-63G $>$ A mediante reacción en cadena de la polimerasa en tiempo real

\begin{tabular}{|c|c|c|}
\hline Polimorfismos & $\begin{array}{c}\text { Sonda Taqman } \\
\text { (Applied Biosystems) }\end{array}$ & Secuencia 5'-3' (VIC/FAM) \\
\hline TERT-1327C >T (rs2735340) & C_1839086_10 & TCGTCTTGTAAATACTTAGGATTAC(A/G)GGTCGCTCTTCTAGAAATCCCCTT \\
\hline TERC-63G>A (rs2293607) & C_16184740_10 & AGGTTTGGGGGTTCACAAGCCCCCA(C/T)TGCCGGCGAGGGGTGACGGAT \\
\hline
\end{tabular}

umbral predefinido, se utilizó para calcular el número de copias repetidas de telómeros con respecto a la proporción del número de copias del gen de una sola copia (T/S) utilizando T/S = $22 \mathrm{DCt}$. La longitud relativa de los telómeros se determinó normalizando la relación (T/S) de cada muestra al ADN calibrador para estandarizar los valores de las muestras en todas las placas de reacción. Para facilitar el manejo de los datos se utilizó el logaritmo natural de este valor ${ }^{22}$.

\section{Análisis de polimorfismos de la telomerasa}

Los polimorfismos del ADN del gen de la telomerasa se midieron mediante un procedimiento de discriminación alélica por PCR en tiempo real realizado en un volumen total de $12 \mu$ l por reacción. Se colocó ADN $(2.5 \mu l)$ en una mezcla de reacción de $9.5 \mu \mathrm{l}$ que contenía $0.25 \mu \mathrm{l}$ de cebadores oligonucleotídicos y sondas Taqman (VIC/FAM), $5 \mu$ l de TaqMan ${ }^{\circledR}$ Universal PCR Master Mix No AmpErase ${ }^{\circledR}$ UNG (Applied Biosystems) y agua bidestilada hasta un volumen final de $12 \mu \mathrm{l}$. Siguiendo las instrucciones del fabricante para el sistema de PCR en tiempo real StepOne Plus (Applied Biosystems), el protocolo de ciclos consistió en un paso de desnaturalización inicial de 10 minutos a $95^{\circ} \mathrm{C}$, seguido de 40 ciclos de desnaturalización a $95^{\circ} \mathrm{C}$ durante 15 segundos, alineamiento a $65^{\circ} \mathrm{C}$ durante 60 segundos y una etapa de extensión a $60^{\circ} \mathrm{C}$ durante 30 segundos.

Las secuencias de las sondas VIC y FAM utilizadas para la detección de polimorfismos se detallan en la tabla 2.

\section{Análisis estadístico}

El análisis estadístico se realizó con el software SPSS Statistics para Windows, versión 2.0, y el programa GenEx 5.3.6 desarrollado para calcular la longitud de los telómeros. Para confirmar los resultados del software GenEx se aplicó la prueba $U$ de Mann Whitney.
Las asociaciones entre la longitud de los telómeros de los leucocitos y los polimorfismos (SNP) de TERT/TERC se modelaron utilizando la prueba de ji al cuadrado

Debido a que algunos datos siguieron una distribución normal y otros (edad) mostraron una distribucición no paramétrica, se utilizó el análisis de la variānza (ANOVA) o la prueba U de Mann Whitney para explórar la influencia de posibles variables de confusión (diäbetes, tabaquismo, hipertensión y edad) sobre la longitud de los telómeros en los pacientes con catarata. También se realizó un análisis de varianza multivariàdo (MANOVA) para comparar estas variables dependientes juntas (diabetes, tabaquismo, hipertensión y edad) en los mismos grupos.

\section{Resultados}

Se encontraron diferencias significativas en la longitud relativa de los telómeros entre los sujetos con catarătas y los que no tenían cataratas independientemente de la edad $(p<0.01)$. Como era de esperar de acuerdo con el impacto de la edad en la longitud de los telómeros, los sujetos sin catarata $\geq 60$ años tenían telómeros más cortos que los sujetos $\leq 60$ años $(p<0.01)$. Sin embargo, se observaron diferencias significativas $(p<0.01)$ en la longitud de los telómeros en sujetos con catarată no relacionada con la edad. Curiosamente, los sujetos más jóvenes con cataratas tenían telómeros más cortosque las personas mayores con catarata (Tabla 3).

La hipótesis de que las variaciones individuales en los genes TERT y TERC de la telomerasa podrían èstar asociadas con una menor actividad de la telomerầsa, y por lo tanto con una menor longitud de los telómeros leucocitarios, no se confirmó en ningún grupo de sujetos incluidos en el estudio (Tabla 4).

El análisis revela que la relación entre la longitud de los telómeros y las cataratas no se vio afectada por factores de riesgo cardiovascular, como la diabetes, el tabaquismo y la hipertensión, en todos los grupos?

En los pacientes con cataratas, el MANOVA no indicó diferencias significativas $(p>0.05)$ en la longitud de 
Tabla 3. Comparación de las longitudes de los telómeros de leucocitos entre los grupos de estudio

\begin{tabular}{|l|c|c|}
\hline & Longitud del telómero & $p$ \\
\hline $\begin{array}{l}\text { Casos vs. } \\
\text { controles }\end{array}$ & $2.88 \pm 1.43$ & $<0.01$ \\
\hline $\begin{array}{l}\text { Casos } \leq 55 \text { años vs. } \\
\text { controles } \leq 55 \text { años }\end{array}$ & $2.17 \pm 1.58$ & \\
\hline $\begin{array}{l}\text { Casos } \geq 60 \text { años vs. } \\
\text { controles } \geq 60 \text { años }\end{array}$ & $3.85 \pm 1.56$ & $<0.01$ \\
\hline $\begin{array}{l}\text { Casos } \leq 55 \text { años vs. } \\
\text { casos } \geq 60 \text { años }\end{array}$ & $4.69 \pm 1.17$ & $<0.01$ \\
\hline $\begin{array}{l}\text { Controles } \leq 55 \text { años vs. } \\
\text { controles } \geq 60 \text { años }\end{array}$ & $2.36 \pm 1.56$ & $<0.01$ \\
\hline
\end{tabular}

Tabla 4. Frecuencias de los genotipos de SNP de TERT y TERC en casos y controles

\begin{tabular}{|l|l|c|c|c|}
\hline SNP & Genotipo & $\begin{array}{c}\text { Casos } \\
\text { n (\%) }\end{array}$ & $\begin{array}{c}\text { Controles } \\
\text { n (\%) }\end{array}$ & p \\
\hline TERT & TT & $31(3.4 \%)$ & $26(25.2 \%)$ & 0.7 \\
rs2735940 & TC & $54(52.9 \%)$ & $58(56.3 \%)$ & 0.44 \\
& CC & $17(16.7 \%)$ & $19(18.4 \%)$ & 0.44 \\
& TT & $31(3.4 \%)$ & $26(25.2 \%)$ & \\
& TC + CC & $71(69.9 \%)$ & $77(74.8 \%)$ & \\
& TT + TC & $85(83.3 \%)$ & $84(81.55 \%)$ & \\
\hline TERC & CC & $17(16.7 \%)$ & $19(18.4 \%)$ & \\
rs2293607 & AA & $60(58.8 \%)$ & $69(67.7 \%)$ & 0.38 \\
& AG & $38(37.3 \%)$ & $29(28.2 \%)$ & 0.25 \\
& GG & $4(3.9 \%)$ & $5(4.9 \%)$ & 1 \\
& AA & $60(58.8 \%)$ & $69(67 \%)$ & \\
& AG + GG & $42(41.2 \%)$ & $34(33 \%)$ & \\
& AG + AA & $98(96.1 \%)$ & $98(95.1 \%)$ & \\
& GG & $4(3.9 \%)$ & $5(4.9 \%)$ & \\
\hline
\end{tabular}

A: adenina; C: citosina; G: guanina; SNP: polimorfismo de un solo nucleótido; T: timina; TERC: componente de ARN de telomerasa; TERT: transcriptasa inversa de telomerasa.

los telómeros leucocitarios en relación con los factores hipertensión, diabetes, tabaquismo y edad. El valor de p obtenido en la prueba ANOVA para cada uno de estos factores de riesgo cardiovascular fue $>0.05$.

Sin embargo, como la edad no sigue una distribución paramétrica, se utilizó la prueba $U$ de Mann Whitney y entonces se observó un efecto significativo de la edad sobre la longitud de los telómeros de los leucocitos $(p<0.05)$.

\section{Discusión}

El desarrollo de cataratas se caracteriza por la senescencia de las células del cristalino. Este envejecimiento es acelerado por el estrés oxidativo y la radiación no ionizante en forma de luz ultravioleta, que induce daño al ADN producido por los radicales libres derivados̄idel oxígeno. Debido a la ausencia de actividad de la tellomerasa en el cristalino, el ataque oxidativo de los hịdroperóxidos de fosfolípidos presentes en el humor acưoso y el daño a las estructuras de la membrana de las células del cristalino probablemente produzcan un acortamiento de los telómeros $1,6,23-25$. Por lo tanto- el aumento del estrés oxidativo y la falta de desintoxicación reductora de los hidroperóxidos de fosfolípidos son los mecanismos desencadenantes del acortamiento de los telómeros y del desarrollo de cataratas.

En este estudio, una mayor transparencia del crístalino se relacionó con una mayor longitud de los têlómeros leucocitarios, como ya han informado otros autores $6,13,23$.

Hasta donde sabemos, el presente estudio es eÉprimero en examinar la longitud de los telómeros de=los leucocitos en pacientes jóvenes con catarata, y en informar longitudes relativamente cortas para el rango de edad. Se observó que las personas más jóvenesçon catarata tenían telómeros más cortos que los contrôles de la misma edad sin cataratas. Además, este gưupo de pacientes también mostró una longitud telomérica más corta que los sujetos mayores con cataratas.

Especulamos que este hallazgo podría reflejab un envejecimiento prematuro en esta población. En č̄onsecuencia, la aparición de cataratas en adultos jóvenes puede servir como un marcador in vivo del envejecimiento natural, y también podría advertir sobre otras afecciones asociadas con el acortamiento y el envejecimiento de los telómeros, como glaucoma, degeneración macular relacionada con la edad ${ }^{24}$, enfermedad cardiovascular o cáncer. Por tanto, los telómēros podrían considerarse nuevos biomarcadores del eñvejecimiento y también predictores del riesgo de determinadas enfermedades degenerativas relacionadas con la edad. Por consiguiente, la longitud de los telómeros podría ayudar a monitorear la eficacia de la preveneión 0 de las estrategias destinadas a modificar el proceso del envejecimiento.

El cristalino tiene varias ventajas reales como $\overline{\text { mar- }}$ cador del envejecimiento. Por ejemplo, utilizando técnicas de imagen rápidas, no invasivas y no peligroš as, se puede medir su transparencia en una escala continua. Además, el uso de un sistema de clasificación como LOCS III ofrece una forma reproducible y precisa de determinar la transparencia del cristalino.

Nuestros resultados también revelaron que los poolimorfismos de los genes TERT y TERC, rs2735940 y rs2293607, no se asociaron con un acortamiento de 
los telómeros en ningún grupo, incluido el de personas más jóvenes con cataratas. Esto significa que el acortamiento de los telómeros observado en los jóvenes con catarata no se asoció con estos polimorfismos de un solo nucleótido (SNP).

Existen varios informes ${ }^{7-11}$ sobre la relación entre la longitud corta de los telómeros y los factores de riesgo cardiovascular, como la hipertensión, la diabetes y el tabaquismo. Analizamos la relación entre posibles factores de confusión (diabetes, hipertensión y tabaquismo) y la longitud de los telómeros leucocitarios. Los participantes de nuestro estudio fueron estratificados por edad, sexo y enfermedad cardiovascular, como se muestra en la tabla 1. En los pacientes con catarata, el análisis multivariado no mostró ninguna relación entre la longitud de los telómeros, la edad y los factores de riesgo cardiovascular. Esto indica que el acortamiento de los telómeros en los pacientes jóvenes con catarata no estuvo influenciado por factores de riesgo cardiovascular ni por polimorfismos de la telomerasa, por lo que este acortamiento podría estar directamente asociado a la catarata. Se necesitan estudios longitudinales para confirmar nuestra hipótesis y fortalecer la idea de que la catarata temprana podría indicar un envejecimiento prematuro y la posible presencia de enfermedades relacionadas con la edad.

Se deben considerar varias limitaciones en la interpretación de nuestros resultados. La longitud de los telómeros en los leucocitos periféricos podría no reflejar alteraciones en la longitud de los telómeros en las células del cristalino, aunque varios estudios han demostrado su buena correlación $n^{12,13,25}$. Además, como queríamos examinar una cohorte de sujetos representativa de la población general, no se excluyó a las mujeres. Sin embargo, es bien sabido que las mujeres adultas tienen una longitud de los telómeros leucocitarios más larga que los hombres ${ }^{26}$, aunque la longitud de los telómeros al nacer es equivalente en niños y niñas ${ }^{12,27}$. Otra limitación es que el tabaquismo acelera el acortamiento de los telómeros y esto se ha relacionado con múltiples resultados negativos para la salud durante la vida de una persona ${ }^{28}$. Algunos estudios han encontrado una relación dosis-respuesta entre la exposición acumulada de por vida al tabaquismo y la longitud de los telómeros en adultos ${ }^{29}$. No obstante, para evitar estos factores de confusión, equilibramos a los fumadores en todos los grupos incluidos en nuestro estudio.

Finalmente, se deben considerar otros factores, como el complejo shelterina ${ }^{30,31}$. Los telómeros consisten en repeticiones en tándem de una secuencia de ADN conectada a seis componentes proteicos conocidos como shelterina ${ }^{30}$, siendo el factor $T$ de unión a las repeticiones teloméricas (TRF1) y la pproteína homóloga de la displasia adrenocortical (TPP 1 ) las proteínas más relevantes en los mamíferos. ¿as proteínas shelterina están especializadas en proteger a los telómeros de los mecanismos de reparación del ADN, así como en regular la actividad de la teloomerasa. Por consiguiente, la supresión de TRF1 y TPP1 da como resultado el desarrollo de enfermedâdes degenerativas y relacionadas con la edad, tales como disqueratosis congénita, anemia aplásica y fibrösis pulmonar idiopática en ratones jóvenes. Estas enffermedades están asociadas con telómeros cortos o disfuncionales y con una falla característica en la capacidad regenerativa de los tejidos ${ }^{31}$. En consecuencia, existen otros complejos, como la shelterina, además de los polimorfismos de la telomerasa, que actúan sobre esta enzima condicionando la longitud del.telómero, y que por lo tanto podrían participar en la âparición de enfermedades relacionadas con la edad, como las cataratas.

Si bien es difícil reclutar pacientes jóvenes con catarata, la relación observada aquí entre una longitud telomérica corta y una catarata prematura requîere confirmación en series más amplias de pacientes

En conclusión, informamos por primera vez que las personas jóvenes con cataratas tienen una Fongitud telomérica de los leucocitos más corta quelas personas sin cataratas, y que esta relación no se ve afectada por factores de riesgo cardiovascular nippor polimorfismos de la telomerasa. Si esta relación se confirma en series más amplias de pacientes, płanteamos la hipótesis de que el desarrollo temprano de cataratas y la longitud corta de los telómeros podrían ser un marcador de envejecimiènto prematuro.

\section{Financiamiento}

Programa de investigación en salud del Institutê⿳㇒ de Salud Carlos III (Pl13/01741) cofinanciado con fondos FEDER. La organización financiadora no tuvo ningún papel en el diseño ni en la realización de ěsta investigación.

\section{Conflicto de intereses}

Los autores declaran que no existen conflictos de intereses. 


\section{Responsabilidades éticas}

Protección de personas y animales. Los autores declaran que los procedimientos seguidos se conformaron a las normas éticas del comité de experimentación humana responsable y de acuerdo con la Asociación Médica Mundial y la Declaración de Helsinki.

Confidencialidad de los datos. Los autores declaran que han seguido los protocolos de su centro de trabajo sobre la publicación de datos de pacientes.

Derecho a la privacidad y consentimiento informado. Los autores han obtenido el consentimiento informado de los pacientes y/o sujetos referidos en el artículo. Este documento obra en poder del autor de correspondencia.

\section{Bibliografía}

1. Von Zglinicki T. Will your telomeres tell your futures? BMJ. 2012;344:e1727.

2. De Rooij SR, Roseboom TJ. The developmental origins of ageing: study protocol for the Dutch famine birth cohort study on ageing. BMJ Open. 2013;3:e003167.

3. Calado RT, Young NS.Telomere diseases. N Engl J Med. 2009;361:2353-65

4. Njajou OT, Blackburn EH, Pawlikowska L, Mangino M, Damcott CM, Kwoket $P$, et al. A common variant in the telomerase RNA component is associated with short telomere length. PLoS One. 2010;5:e13048.

5. Matsubara Y, Murata M, Yoshida T, et al. Telomere length of normal leukocytes is affected by a functional polymorphism of hTERT. Biochem Biophys Res Commun. 2006;341:128-31.

6. Von Zglinicki T, Martin-Ruiz CM. Telomeres as biomarkers for ageing and age-related disease. Curr Mol Med. 2005;5:197-203.

7. Zee RY, Castonguay AJ, Barton NS, et al. Mean leukocyte telomere length shortening and type 2 diabetes mellitus: a case-control study. Transl Res. 2010;155:166-9.

8. Testa R, Olivieri F, Sirolla C, et al. Leukocyte telomere length is associated with complications of type 2 diabetes mellitus. Diabet Med. 2011;28:1388-94.

9. Babizhayev MA, Yegorov YE. Smoking and health: association between telomere length and factors impacting on human disease, quality of life and life span in a large population-based cohort under the effect of smoking duration. Fundam Clin Pharmacol. 2011;25:425-42.

10. Demissie S, Levy D, Benjamin EJ, et al. Insulin resistance, oxidative stress, hypertension, and leukocyte telomere length in men from the Framingham Heart Study. Aging Cell. 2006;5:325-30.
11. Fitzpatrick AL, Kronmal RA, Gardner JP, et al. Leukocyte telomere length and cardiovascular disease in the cardiovascular health study. Am J Epidemiol. 2007;165:14-21.

12. Aviv A, Valdes AM, Spector TD. Human telomere biology: pitfalls of moving from the laboratory to epidemiology. Int J Epidemiol. 2006;35:1424-9.

13. Sanders JL, lannaccone A, Boudreau RM, et al. The association of cataract with leukocyte telomere length in older adults: defining asnew marker of aging. J Gerontol A Biol Sci Med Sci. 2011;66:639-45. ह

14. Dollfus $H$, Porto $P$, Caussade $P$, et al. Ocular manifestations in theyinherited DNA repair disorders. Surv Ophthalmol. 2003;48:107-22.

15. Kipling D, Davis T, Ostler ET, et al. What can progeroid syndrome tell us about human aging? Science. 2004;305:1426-31.

16. Gomez-Sanchez JC, Delgado-Esteban M, Sobrino T, et al. The hüman Tp53 Arg72Pro polymorphism explains different functional prognessis in stroke. J Exp Med. 2011;208:429-37.

17. Gil ME, Coetzer TL. Real-time quantitative PCR of telomere length Mol Biotechnol. 2004;27:169-72.

18. Cawthon RM. Telomere measurement by quantitative PCR. Nucleic Äcids Res. 2002;30:e47.

19. Perez-Rivera JA, Pabon-Osuna P, Cieza-Borrella C, et al. Prognostic value of telomere length in acute coronary syndrome. Mech Ageing Dev. 2012;133:695-7.

20. Perez-Rivera JA, Pabon-Osuna P, Cieza-Borrella C, et al. Effect oftelomere length on prognosis in men with acute coronary syndrome. Am J Cardiol. 2014;113:418-21.

21. Martinez-Delgado B, Yanowsky K, Inglada-Perez L, et al. Genetic anticipation is associated with telomere shortening in hereditary breast cancer. PLoS Genet. 2011;7:e1002182.

22. Killick E, Tymrakiewicz M, Cieza-Borrella C, et al. Telomere length shows no association with BRCA1 and BRCA2 mutation status. PLOSOne. 2014;9:e86659.

23. Berthoud VM, Beyer EC. Oxidative stress, lens gap junctions, andَّataracts. Antioxid Redox Signal. 2009;11:339-53.

24. Weng $X$, Zhang $H$, Kan M, et al. Leukocyte telomere length is assoejated with advanced age-related macular degeneration in the Han Chinese population. Exp Gerontol. 2015;69:36-40.

25. Babizhayev MA, Vishnyakova KS, Yegorov YE. Telomere-dependent senescent phenotype of lens epithelial cells as a biological marker of aging and cataractogenesis: the role of oxidative stress intensity and specific mechanism of phospholipid hydroperoxide toxicity in lens and aqueous. Fundam Clin Pharmacol. 2010;25:139-62.

26. Benetos A, Okuda K, Lajemi M, et al. Telomere length as an indicator of biological aging: the gender effect and relation with pulse pressute and pulse wave velocity. Hypertension. 2001;37:381-5.

27. Okuda K, Bardeguez A, Garner JP, et al. Telomere length in the new⿳亠丷厂 Pediatr Res. 2002;52:377-81.

28. Weischer M, Bojesen SE, Nordestgaard BG. Telomere shorting unrelated to smoking, body, weight, physical activity, and alcohol intake: $\mathbf{4 , 5 7 6}$ general population individuals with repeat measurements 10 years apart. Plos Genetics. 2014;10:e1004191.

29. Theall KP, Mckasson S, Mabile E, et al. Early hits and long-term consequences: tracking the lasting impact of prenatal smoke exposure on telomere length in children. Am J Pub Heatlh. 2013;103:133-5.

30. García-Beccaria M, Martínez P, Flores JM, et al. In vivo role of checkpoint kinase 2 in signaling telomere dysfunction. Aging Cell. 2014;13:810-6.

31. Donate LE, Blasco MA. Telomeres in cancer and ageing. Phil Trans $R$ Soc B. 2011;366:76-84. 